Research Paper

\title{
Low-Frequency Electrical Stimulation of Denervated Skeletal Muscle Retards Muscle and Trabecular Bone Loss in Aged Rats
}

Hiroyuki Tamaki ${ }^{1}{ }^{\circledR}$, Kengo Yotani ${ }^{2}$, Futoshi Ogita ${ }^{2}$, Keishi Hayao $^{1}$, Hikari Kirimto $^{3}$, Hideaki Onishi ${ }^{1}$, Norikatsu Kasuga4, Noriaki Yamamoto1,5

1. Institute for Human Movement and Medical Sciences, Niigata University of Health and Welfare, Japan

2. Department of Sports and Life Science, National Institute of Fitness and Sports in Kanoya, Japan

3. Department of Sensorimotor Neuroscience, Hiroshima University, Japan

4. Aichi University of Education, Japan

5. Niigata Rehabilitation Hospital, Japan

$\triangle$ Corresponding author: Hiroyuki Tamaki, Ph.D., National Institute of Fitness and Sports in Kanoya, 1 Shiromizu, Kanoya, 981-2393, Japan. E-mail: tamaki@nifs-k.ac.jp

(C) Ivyspring International Publisher. This is an open access article distributed under the terms of the Creative Commons Attribution (CC BY-NC) license (https://creativecommons.org/licenses/by-nc/4.0/). See http://ivyspring.com/terms for full terms and conditions.

Received: 2018.12.26; Accepted: 2019.05.02; Published: 2019.06.02

\begin{abstract}
Electrical stimulation (ES)-induced muscle contraction has multiple effects; however, mechano-responsiveness of bone tissue declines with age. Here, we investigated whether daily low-frequency ES-induced muscle contraction treatment reduces muscle and bone loss and ameliorates bone fragility in early-stage disuse musculoskeletal atrophy in aged rats. Twenty-seven-month-old male rats were assigned to age-matched groups comprising the control (CON), sciatic nerve denervation (DN), or DN with direct low-frequency ES (DN+ES) groups. The structural and mechanical properties of the trabecular and cortical bone of the tibiae, and the morphological and functional properties of the tibialis anterior (TA) muscles were assessed one week after DN. ES-induced muscle contraction force mitigated denervation-induced muscle and trabecular bone loss and deterioration of the mechanical properties of the tibia mid-diaphysis, such as the stiffness, but not the maximal load, in aged rats. The TA muscle in the DN+ES group showed significant improvement in the myofiber cross-sectional area and muscle force relative to the DN group. These results suggest that low-frequency ES-induced muscle contraction treatment retards trabecular bone and muscle loss in aged rats in early-stage disuse musculoskeletal atrophy, and has beneficial effects on the functional properties of denervated skeletal muscle.
\end{abstract}

Key words: disuse, osteopenia, muscle force, micro computed tomography

\section{Introduction}

Bone atrophy is characterized by a reduction in muscle and bone volume and bone mineral density (BMD) and alterations in trabecular and cortical bone architecture. Age-related and disuse-induced alterations to structural and material properties result in decreased mechanical bone strength and an increased risk of bone fracture. Electrical stimulation (ES) has been utilized for patients as a therapeutic intervention and a functional substitute for voluntary muscle contractions, and has been explored as a means of counteracting the skeletal muscle atrophy that occurs as a result of various clinical conditions, such as spinal cord injury, aging, and disuse $[1,2]$. In experimental animals, ES has been shown to help limit DN-induced muscle atrophy and improve muscle force and recovery. The skeletal muscles of aged rats also have a greater capability for maintaining mass and force generation [3]. However, other studies have generated contradictory results [4, 5]. Thus, the effects of ES on skeletal muscles may depend on the atrophy model and the ES parameters, particularly the intensity, frequency and duration of stimulation. We previously assessed beneficial stimulation intensities and frequencies of ES, based on the structural recovery of individual skeletal muscles after DN, and found that direct ES with $16 \mathrm{~mA}$ at 10 
$\mathrm{Hz}$ retarded denervated muscle atrophy and upregulated the mRNA expression of insulin-like growth factor-1 (IGF-1) [6].

The maintenance of muscle volume is also a significant factor in maintaining bone volume and strength, because muscles are responsible for generating the force that drives beneficial mechanical stress on bone in vivo, in the form of physical exercise. In a disuse animal model, frequency-dependent muscle stimulation $(20-100 \mathrm{~Hz})$ for four weeks inhibited trabecular bone loss in the disused rat femur [7]. Recent studies using low-frequency ES at $10 \mathrm{~Hz}$ (incomplete tetanus) have reported delayed trabecular bone and muscle loss during the early stages of musculoskeletal atrophy in denervated rats $[8,9]$. In addition, the positive effects of $10 \mathrm{~Hz}$ ES-induced muscle contraction on trabecular bone and bone strength may be partly due to the activation of mechanosensors and the dentin matrix protein 1 (DMP1) production of osteocytes in bone tissue in young rats $[9,10]$. Osteocytes are the principal mechanosensory cells in bone tissue, and aging and disuse contribute to increases in osteocyte apoptosis and decreases in osteocyte density [11]. Thus, bone in adult and aged rats appears to be less sensitive to mechanical loading than the growing bone of young rats.

Compared with young adult rats, the bones of aged rats have an equal or greater cortical area and width, with a lower trabecular bone volume, thickness, and BMD [12]. These changes in geometry and material properties may affect bone mechanical properties, and the effects of hind limb disuse in adult rats reportedly occur more slowly at the tissue level. The mechano-responsiveness of the periosteal and endocortical surfaces are significantly smaller in adult and aged tibiae than in young tibiae [13]. Furthermore, substantial structural and functional decreases occur in the number of motor units, and the number of denervated fibers in skeletal muscles increases with age. The increased number of denervated muscle fibers in aged age results in a decreased contractile ability to generate the muscle force that induces mechanical bone loading. Therefore, in light of these previous studies that have reported differences in bone and skeletal muscle tissue between young and aged rats, little is known about whether ES-induced muscle contraction treatment would also be beneficial in skeletally-mature, aged rats.

Furthermore, muscle and trabecular bone loss and their morphological changes commence in the first week after denervation. Reportedly, early therapeutic intervention effectively attenuates the loss of muscle mass and bone strength [10]. Thus, these reports support the concept of early therapeutic intervention to ameliorate muscle and bone atrophy and the associated potentially irreversible deterioration of trabecular architecture in the early stages of disuse. We hypothesized that daily low-frequency ES-induced muscle force treatment would effectively reduce trabecular bone loss, providing that a certain amount of muscle mass to function as a force generator is retained in the early stages of disuse atrophy in aged rats. Yet, it remains unclear whether this type of ES treatment would have a positive effect on the mechanical properties of cortical bone and its determinant factors in early-stage disuse atrophy in aged rats. Thus, the aim of this study was to investigate whether the daily low-frequency ES-induced muscle contraction treatment that reduces muscle and bone loss and ameliorates bone fragility, in early-stage disuse musculoskeletal atrophy in young rats, would also have beneficial effects in reducing these properties in the denervated TA muscles and tibiae of aged rats. The mechanical properties of the tibia cortical bone in relation to maximal load, stiffness, and elastic modulus were also assessed.

\section{Materials and Methods}

\section{Animals and experimental protocol}

Twenty-four male Fischer 344 rats (CLEA, Tokyo, Japan) were housed in standard cages under a constant temperature $\left(23 \pm 2{ }^{\circ} \mathrm{C}\right)$, humidity $(55 \% \pm 5 \%)$, and in $12 \mathrm{~h}$ light-dark cycles. The rats had free access to CE-2 rodent chow (CLEA) and water. At 27 months of age, the rats were randomly assigned to one of the following groups: the age-matched control group $(\mathrm{CON})$; the sciatic denervation group $(\mathrm{DN})$; or the $\mathrm{DN}$ + direct electrical stimulation group (DN+ES). The sample size ( $\mathrm{n}=8$ /group) was calculated with reference to trabecular bone fraction $(\mathrm{BV} / \mathrm{TV})$ data from previous studies in our laboratory, using the following formula $[10,14]$ :

$$
n=(\mathrm{r}+1) / \mathrm{r} \times \sigma^{2}\left(\mathrm{Z}_{\alpha / 2}+\mathrm{Z}_{\beta}\right)^{2} / \Delta^{2},(1)
$$

where $r$ is the ratio of the larger group to the smaller group, $\sigma$ is the standard deviation, $\Delta$ is the effect size, $\alpha=0.05, \beta=0.2$ (for $80 \%$ power with $95 \%$ confidence), $Z_{\alpha / 2}=1.96$, and $Z_{\beta}=0.84$.

The rats in the DN groups were anesthetized by inhalation of $2 \%-2.5 \%$ isoflurane in air with a flow of $2 \mathrm{~L} /$ minute. An incision was made at the skin covering the left buttock, and the sciatic nerve was exposed, then frozen with a stainless steel rod cooled in liquid nitrogen $[8,10,15]$. The incision was then closed with sutures, and each animal was kept in a standard cage. A complete lack of active movement at the ankle and toe was observed on the denervated 
side after nerve freezing. To confirm the efficacy of denervation, evoked electromyography (EMG) was recorded from the denervated tibialis anterior (TA) muscle with ES at a location proximal to the freezing site of the sciatic nerve at the end of the experiments $[9,10]$. No EMG recordings were observed in the denervated TA (Figure 1). The rats in the ES groups were administered with direct muscle ES for one week, commencing the day after DN surgery. All procedures were approved by the Animal Committee of the National Institute of Fitness and Sports and the Animal Committee of Niigata University of Health and Welfare.

\section{Direct ES procedures and evoked muscle contraction force measurement}

The stimulation protocol was delivered as described previously [8-10]. The day after surgery, the left TA muscles of the DN+ES rats under isoflurane inhalation anesthesia $(2 \%-2.5 \%)$ were percutaneously electrically stimulated. Bipolar silver surface electrodes ( $3 \mathrm{~mm}$ diameter) were attached to the shaved anterior surface of the rat's left leg. Direct muscle stimulation was applied using an electrostimulator and isolator (SEM-4201, SS-201, Nihon Kohden, Tokyo, Japan) for 30 minutes a day, 6 days a week, for 1 week, at an intensity of $16 \mathrm{~mA}$, with a $10 \mathrm{~Hz}$ frequency, and a pulse width of $250 \mu$ s. The ES regimen was carried out with $2 \mathrm{~s}$ of stimulation followed by a $6 \mathrm{~s}$ rest. Although this did not cause a maximal contraction [8] in denervated TA muscle, it evoked visible toe flexion. The rats in the CON and DN group were also anesthetized with isoflurane inhalation $(2 \%-2.5 \%)$ for the same time period as the ES rats. One week after denervation, the isometric contraction force in TA muscle was measured under the same stimulus conditions as the daily ES regimen to determine the mechanical factors evoked by direct ES, as previously described $(n=8 /$ group) $[8,9]$. Briefly, the lower limbs of rats under inhalational anesthesia were secured and stabilized on the working platform with restraining bars and pins at the knee and ankle joints. The distal tendon of the TA was attached to an isometric transducer (TB-654T, Nihon Kohden) that was secured with a 4-0 silk suture on a three-dimensional (3D) drive precision stage. The muscle tension signal was sampled at $2 \mathrm{kHz}$ through a PowerLab 8SP A/D converter (ADInstruments, Nagoya, Japan).

\section{Bone strain measurements}

To check the tibia strains during ES of the same stimulus condition as the daily ES regimen, strain gages (KFG-3-120, Kyowa, Tokyo, Japan) were attached in longitudinal alignment to the center of medial surface at the proximal/middle site of right tibiae of four rats, under isoflurane inhalation anesthesia. After removing the soft tissue on the bonding site, the bone surface was degraded, dried and the gage was bonded with cyanoacrylate instantaneous adhesive (CC-33A, Kyowa) pressing the strain gage covered with the polyethylene sheet for approximately 1 minute. Strain signals were processed using an amplifier (Four Assist, Tokyo, Japan) and data were collected at $2 \mathrm{kHz}$ with a PowerLab 8SP A/D converter (ADInstruments). Peak strains and maximum and minimum strain rates during ES treatment were measured using Chart 7 software (ADInstruments).

\section{Tissue preparation}

The tibiae and TA muscles were harvested from rats anesthetized with sodium pentobarbital (50 $\mathrm{mg} / \mathrm{kg}$, i.p.) at the end of the experiment. The TA muscles were weighed, and samples were mounted on a piece of cork with OCT compound and frozen in isopentane cooled in liquid nitrogen for histological analyses. Samples were stored at $-80^{\circ} \mathrm{C}$ until use. TA muscle weight (MW) was normalized by body weight (BW), and is expressed as the ratio of $\mathrm{MW} / \mathrm{BW}$. The tibiae were individually wrapped in saline soaked gauze and stored at $-20^{\circ} \mathrm{C}$ until micro-CT analysis and bone biomechanical testing was performed.

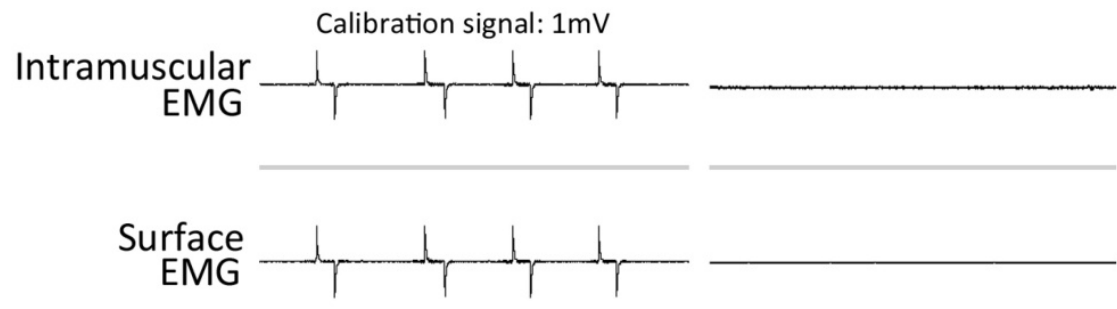

Stimulation signal

Figure 1. Intramuscular and surface EMG recordings from the denervated TA muscles during sciatic nerve stimulation after 1 week of denervation. 


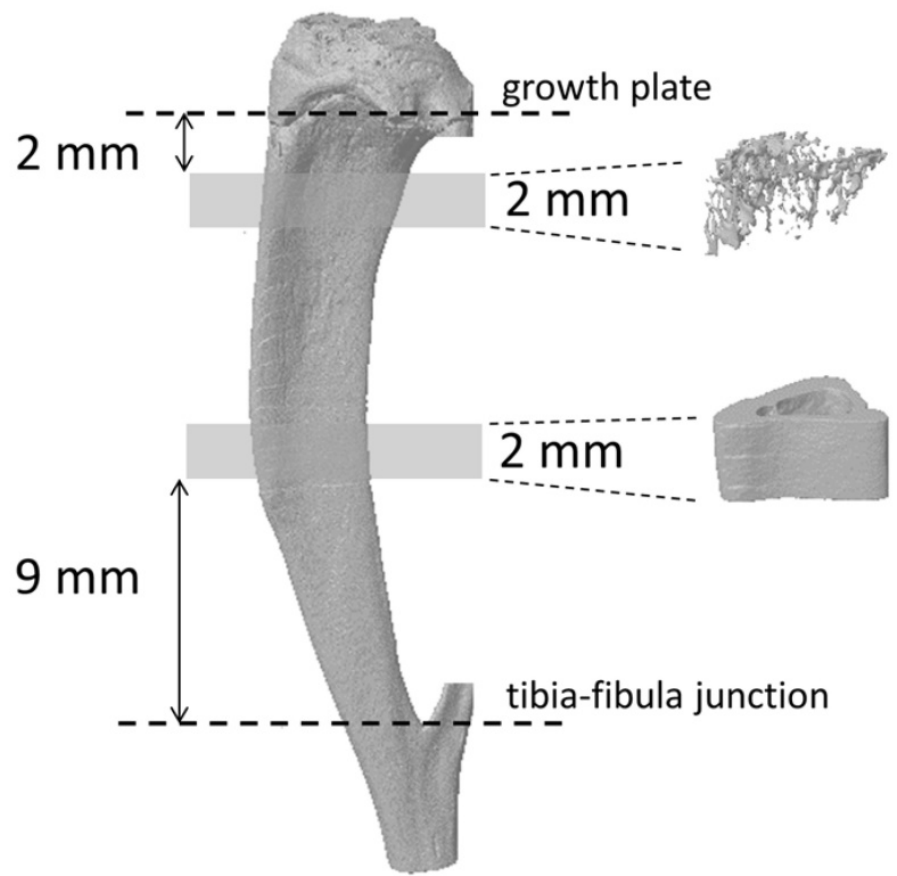

Figure 2. 3D images of the tibia. Trabecular bone at the metaphyseal section and cortical bone at the mid-diaphysis of the tibia were evaluated using micro-CT.

\section{Microcomputed tomography (micro-CT)}

The tibial bone microarchitecture was measured using a SkyScan 1076 high-resolution micro-CT scanner (SkyScan, Kontich, Belgium). Details regarding the micro-CT scanner and analysis software used in this study have been described previously [8-10]. Briefly, tibia bone scanning was performed using a source voltage/current of $70 \mathrm{kV} / 141 \mu \mathrm{A}$, with a $1 \mathrm{~mm}$ aluminum filter to reduce beam hardening. Scans were made with a rotation step of $0.6^{\circ}$ through to $180^{\circ}$ and a pixel size of $17.67 \mu \mathrm{m}$. The 3D microstructural image data were reconstructed and morphometric parameters were calculated using the NRecon and CT Analyzer software (SkyScan, Figure 2). Trabecular bone within the proximal tibiae and cortical bone at the tibial midshaft were extracted by semi-automatically drawing interactive polygons on two-dimensional (2D) sections. The volume of interest (VOI) started at a distance of $2 \mathrm{~mm}$ from the lower end of the growth plate and extended distally for 114 cross sections (height $=2 \mathrm{~mm}$ ). The VOI comprised only trabecular bone and the marrow cavity. To analyze tibial cortical bone, the VOI was positioned in the region starting $9 \mathrm{~mm}$ proximal to the tibia-fibula junction for $2 \mathrm{~mm}$ towards the midshaft. The following parameters were measured according to the guidelines and nomenclature proposed by the American Society for Bone and Mineral Research [16]: trabecular bone volume fraction $(\mathrm{BV} / \mathrm{TV}, \%)$, trabecular number (Tb.N, 1/mm), trabecular thickness (Tb.Th, $\mathrm{mm}$ ), trabecular spacing (Tb.Sp, $\mathrm{mm})$, connection density (Conn.D, $\left.1 / \mathrm{mm}^{3}\right)$, total tissue volume $\left(\mathrm{TV}, \mathrm{mm}^{3}\right)$, cortical bone fraction (Ct.Ar/Tt.Ar, \%), and cortical porosity (Ct.Po, as Po.V/Ct.V, \%). The second moment of area $\left(\mathrm{I}, \mathrm{mm}^{4}\right)$, which is a measure of the efficiency of a cross-sectional shape to resist bending caused by loading, was calculated using the following formula [17]: $I=\Pi / 64 \times\left(D_{1} D_{2}{ }^{3}-d_{1} d_{2}{ }^{3}\right)$, where $D_{1}$ is the right-left (RL) outer diameter $(\mathrm{mm}), \mathrm{D}_{2}$ is the anteroposterior (AP) outer diameter $(\mathrm{mm}), \mathrm{d}_{1}$ is the RL inner diameter $(\mathrm{mm})$, and $\mathrm{d}_{2}$ is the AP inner diameter $(\mathrm{mm})$. The estimated mineral density of the cortical bone tissue was determined based on the linear correlation between the CT attenuation coefficient and bone mineral density (BMD) [18]. To calibrate the volumetric BMD of each bone specimen, two hydroxyapatite phantoms $\left(0.25\right.$ and $0.75 \mathrm{~g} / \mathrm{cm}^{3}$ HA BMD phantoms, SkyScan) were used, following the manufacturer's instructions (bone mineral density calibration in SkyScan CT-analyzer, SkyScan).

\section{Biomechanical testing}

The mechanical properties of the maximum load $(\mathrm{N})$, stiffness $(\mathrm{S} ; \mathrm{N} / \mathrm{mm}$ ), and elastic modulus (Em; $\mathrm{N} / \mathrm{mm}^{2}$ ) of the tibiae were determined by the three-point bending test using an EZ-SX mechanical testing device (Shimadzu, Tokyo, Japan), as described previously [10]. Briefly, the tibia was placed on supports (anterior surface down) that were spaced at a distance of $20 \mathrm{~mm}$, and then loaded to failure at a constant displacement rate of $1 \mathrm{~mm} / \mathrm{min}$. Load-deformation data were recorded on a computer, and the following mechanical parameters were 
calculated from the load-deformation curves using Trapezium X software (Shimadzu). Stiffness was calculated as the slope of the elastic deformation of the bone, i.e., the slope of the linear segment of the force-deformation curve. The elastic modulus was derived as [17] $\mathrm{Em}=1 / 48 \times \mathrm{L}^{3} \times \mathrm{S} / \mathrm{I}$, where $\mathrm{L}$ is the distance between the two supports, and I is the second moment of area.

\section{Immunohistochemistry and image analysis}

The TA muscle samples were cut into $10 \mu \mathrm{m}$ cross sections at $-20^{\circ} \mathrm{C}$ with a cryostat (CM3050S, Leica, Germany) and mounted on silanized slides for immunohistochemical staining. After air-drying at room temperature and fixing with ice-cold $4 \%$ paraformaldehyde for $15 \mathrm{~min}$, sections were blocked at room temperature for $1 \mathrm{~h}$ with $10 \%$ normal goat serum (NGS) and 1\% Triton X-100 in PBS, then washed twice in PBS for 5 min. Next, sections were incubated in 5\% NGS and 0.3\% Triton X-100 in PBS for $16-20 \mathrm{~h}$ at $4^{\circ} \mathrm{C}$ with a primary antibody against laminin as a marker for basement membrane integrity (1:200 dilution, Abcam, Tokyo, Japan). The sections were washed several times with PBS, incubated with an appropriate secondary antibody (Alexa Fluor 568 goat anti-rabbit IgG, 1:500 dilution, Abcam), diluted with PBS containing $5 \%$ NS and $0.1 \%$ Triton X-100 for $1 \mathrm{~h}$ at room temperature, and finally mounted with Vectashield mounting medium. Images of TA muscle sections were obtained using a fluorescent light microscope (BX60; Olympus, Tokyo, Japan) and a charge-coupled device (CCD) camera (DP73; Olympus, Tokyo, Japan). Digital images at 200-fold magnification were used to determine the cross-sectional area of the muscle fibers (FCSA) in each TA muscle. The FCSAs of at least 100 fibers in each muscle were measured using Image-Pro Premier software (Media Cybernetics).

\section{Statistical analysis}

All data are expressed as the mean \pm standard deviation. Data sets were analyzed using one-way analysis of variance (ANOVA) followed by either Tukey's post hoc test or the Kruskal-Wallis test followed by a Steel-Dwass multiple comparisons test (Ekuseru-Toukei 2015 software for Windows, Social Survey Research Information Co., Ltd., Tokyo, Japan), depending on the normality of the data distribution. Significance levels were set at $P<0.05$.

\section{Results}

\section{Muscle weight, ES-evoked muscle contraction force and bone strain}

The TA and soleus (Sol) muscle weights significantly decreased after denervation $(P<0.05)$.
Relative TA muscle weights were significantly lower in the DN group compared with the CON group $(P<$ $0.05)$, and were significantly higher in the DN+ES group compared with the DN group $(P<0.05)$. The relative Sol muscle weight was significantly lower $(P$ $<0.05$ ) in the DN and DN+ES groups than in the CON group, but no significant difference was observed between the DN and DN+ES groups. The cross-sectional area of the TA muscle fibers (FCSA) and the muscle contraction force evoked by direct ES at $10 \mathrm{~Hz}$ were significantly smaller in the DN and $\mathrm{DN}+\mathrm{ES}$ groups than in the CON group, and were also smaller in the DN group compared with the DN+ES group $(P<0.05$, Table 1 , Figure 3$)$. Peak strain of the tibia during direct ES treatment at $10 \mathrm{~Hz}$ was $64 \pm 10$ $\mu \varepsilon$. Maximum and minimum strain rates were $7420 \pm$ $3838 \mu \varepsilon / \mathrm{s}$ and $-5534 \pm 1732 \mu \varepsilon / \mathrm{s}$, respectively.

Table 1. Body weight (BW) and muscle weight (MW) of the tibialis anterior (TA) muscle.

\begin{tabular}{llllll}
\hline & & CON & DN & DN+ES & ANOVA \\
\hline Body weight & $(\mathrm{g})$ & $386 \pm 32$ & $384 \pm 18$ & $368 \pm 22$ & 0.356 \\
TA muscle weight & $(\mathrm{mg})$ & $566 \pm 31$ & $459 \pm 47^{*}$ & $512 \pm 53$ & $<0.001$ \\
Relative muscle weight & $(\mathrm{MW} / \mathrm{BW})$ & $1.47 \pm 0.09$ & $1.19 \pm 0.10^{*}$ & $1.39 \pm 0.13 \dagger$ & $<0.001$ \\
Sol muscle weight & $(\mathrm{mg})$ & $131 \pm 11$ & $96 \pm 11^{*}$ & $92 \pm 13^{*}$ & $<0.001$ \\
Relative muscle weight & $(\mathrm{MW} / \mathrm{BW})$ & $0.34 \pm 0.04$ & $0.25 \pm 0.03^{*}$ & $0.25 \pm 0.05^{*}$ & $<0.001$ \\
TA FCSA & $\left(\mathrm{\mu m}^{2}\right)$ & $1212 \pm 129$ & $828 \pm 122^{*}$ & $1038 \pm 116^{*} \dagger$ & $<0.001$ \\
TA muscle force @10Hz & $(\mathrm{N})$ & $1.55 \pm 0.13$ & $0.98 \pm 0.15^{*}$ & $1.28 \pm 0.24^{*} \dagger$ & $<0.001$ \\
\hline
\end{tabular}

Notes: Values are means $\pm \mathrm{SD}$.

\section{Bone micro-CT analysis}

Figure 4 shows typical 3D reconstructed micro-CT images of the proximal tibial metaphyseal trabecular bone in aged rats. Denervation resulted in a significantly smaller trabecular bone volume fraction $(\mathrm{BV} / \mathrm{TV})$, a reduced trabecular thickness (Tb.Th), trabecular number (Tb.N), and connectivity density (Conn.D), and increased trabecular separation (Tb.Sp) in the DN compared with the CON group $(P<0.05$; Table 2, Figure 4). The BV/TV, Tb.Th, and Tb.N were significantly higher in the DN+ES group than in the DN group $(P<0.05)$, but not the Tb.Sp and Conn.D. Morphological analysis of the tibia mid-diaphysis from micro-CT images showed that the tissue volume, cortical bone fraction, cortical porosity, and second moment of area did not differ between the groups (Table 2). CT-estimated BMD in the metaphyseal cortical bone tissue was 3\% lower in the DN group than in the CON group, and 5\% higher in the DN+ES group than in the DN group. However, we did not find any significant differences in BMD between groups.

\section{Bone biomechanical analysis}

Table 3 shows the results of the mechanical property analysis of the tibia mid-diaphysis (Figure 
5). DN-induced disuse resulted in reduced maximal load $(15 \%, P=0.06)$, stiffness $(24 \%, P<0.05)$, and elastic modulus $(33 \%, P<0.05)$ compared with the CON group. The maximal load $(P=0.13)$, stiffness $(P$ $<0.05)$, and the elastic modulus $(P=0.31)$ were $2 \%$, $23 \%$, and $21 \%$ higher in the DN+ES group than in the DN group, respectively (Table 3), but neither the maximal load nor elastic modulus differed significantly.

Table 2. The 3D microstructural properties of trabecular and mid-tibial diaphyseal cortical bone, and bone mineral density (BMD).

\begin{tabular}{llllll}
\hline & & CON & DN & DN+ES & $\begin{array}{l}P \\
\text { values }\end{array}$ \\
\cline { 1 - 3 } Trabecular bone & & & & & \\
BV/TV & $(\%)$ & $9.9 \pm 1.7$ & $5.4 \pm 1.9^{*}$ & $8.4 \pm 1.6 \dagger$ & $<0.001$ \\
Tb.Th & $(\mathrm{mm})$ & $0.095 \pm 0.013$ & $0.080 \pm 0.009^{*}$ & $0.096 \pm 0.007 \dagger$ & 0.015 \\
Tb.N & $(\# / \mathrm{mm})$ & $1.1 \pm 0.2$ & $0.6 \pm 0.2^{*}$ & $0.9 \pm 0.1 \dagger$ & $<0.001$ \\
Tb.Sp & $(\mathrm{mm})$ & $0.49 \pm 0.08$ & $0.64 \pm 0.10^{*}$ & $0.56 \pm 0.04$ & 0.005 \\
\hline
\end{tabular}

\begin{tabular}{llllll}
\hline & & CON & DN & DN+ES & $\begin{array}{l}P \\
\text { values }\end{array}$ \\
\hline Conn.D & $\left(\# / \mathrm{mm}^{3}\right)$ & $18.1 \pm 6.2$ & $11.3 \pm 4.1^{*}$ & $11.5 \pm 2.1^{*}$ & 0.015 \\
Cortical bone & & & & & \\
Tissue volume & $\left(\mathrm{mm}^{3}\right)$ & $15.2 \pm 0.9$ & $15.4 \pm 1.4$ & $15.3 \pm 1.1$ & 0.950 \\
Bone volume & $\left(\mathrm{mm}^{3}\right)$ & $9.7 \pm 0.6$ & $10.2 \pm 1.0$ & $10.0 \pm 0.7$ & 0.548 \\
Cortical bone & $(\%)$ & $64.0 \pm 2.5$ & $66.1 \pm 4.4$ & $65.4 \pm 3.8$ & 0.529 \\
fraction & & & & & \\
Cortical porosity & $(\%)$ & $0.03 \pm 0.04$ & $0.06 \pm 0.11$ & $0.10 \pm 0.13$ & 0.692 \\
BMD & $\left(\mathrm{mgHA} / \mathrm{cm}^{3}\right)$ & $726 \pm 113$ & $704 \pm 110$ & $740 \pm 117$ & 0.832 \\
\hline Notes: Values are means $\pm \mathrm{SD} .{ }^{*} P<0.05$, versus $\mathrm{CON} .+P<0.05$, versus $\mathrm{DN}$.
\end{tabular}

Table 3. Mechanical properties of the mid-tibial diaphyseal cortical bone.

\begin{tabular}{llllll}
\hline & & CON & DN & DN+ES & $P$ values \\
\hline Maximum Load & $(\mathrm{N})$ & $110 \pm 8$ & $94 \pm 12^{*}$ & $96 \pm 17$ & 0.056 \\
Stiffness & $(\mathrm{N} / \mathrm{mm})$ & $180 \pm 21$ & $137 \pm 18^{*}$ & $168 \pm 28 \dagger$ & 0.005 \\
Elastic modulus & $\left(\mathrm{N} / \mathrm{mm}^{2}\right)$ & $5749 \pm 1295$ & $3870 \pm 1763^{*}$ & $4688 \pm 720$ & 0.048 \\
$\begin{array}{l}\text { Second moment of } \\
\text { area }\end{array}$ & $\left(\mathrm{mm}^{4}\right)$ & $6.6 \pm 0.8$ & $7.2 \pm 1.2$ & $7.1 \pm 1.1$ & 0.575 \\
\hline
\end{tabular}

Notes: Values are means \pm SD. ${ }^{*} P<0.05$, versus $\mathrm{CON} . \dagger P<0.05$, versus DN

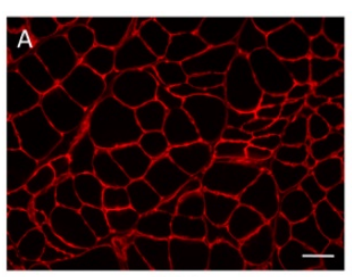

CON

D

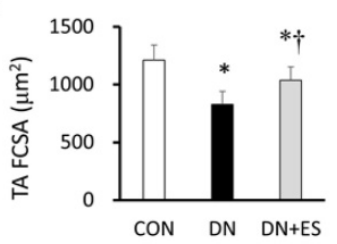

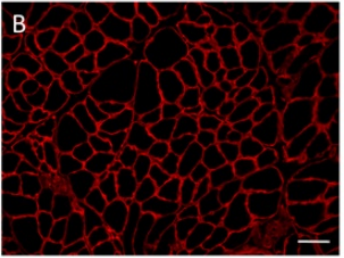

DN

\footnotetext{
E
}

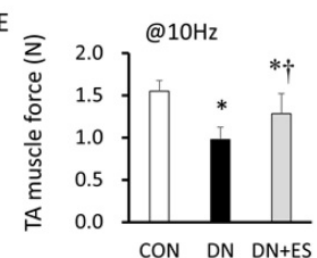

Figure 3. Immunofluorescence photomicrographs of tibialis anterior (TA) muscles for laminin (red) in the CON (A), DN (B), and DN+ES (C) groups, and quantification of mean myofiber cross-sectional area (FCSA) (D) and muscle force $(E)$. Bar $50 \mu \mathrm{m}$. $* P<0.05$ vs. CON; $\uparrow P<0.05$ vs. DN. (CON, control; DN, sciatic nerve denervation; DN+ES, sciatic nerve denervation + electrical stimulation).

\section{A}

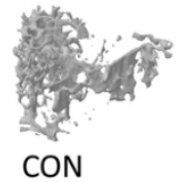

B
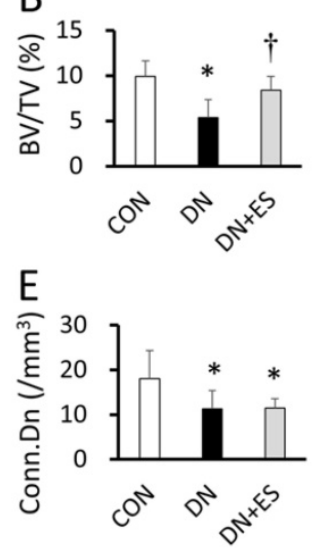

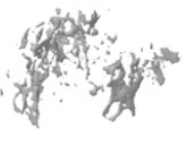

DN

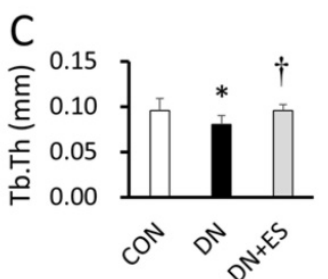

$\mathrm{F}$

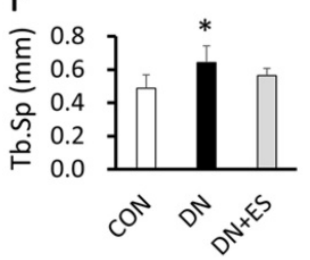

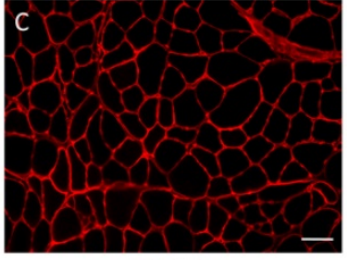

$\mathrm{DN}+\mathrm{ES}$
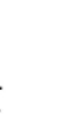


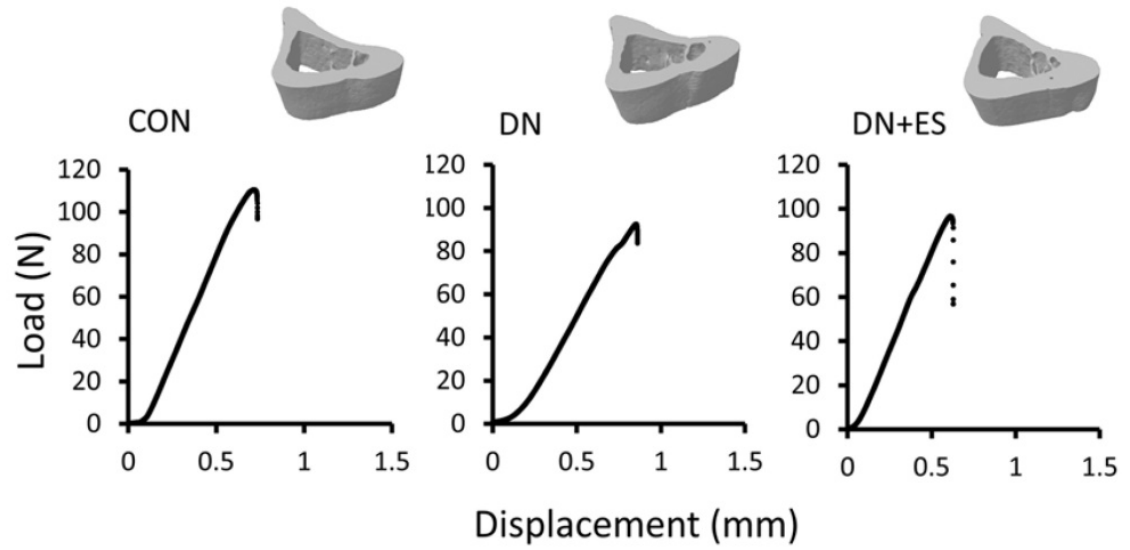

Figure 5. Representative 3D images of the tibia mid-diaphysis from micro-CT (upper panel) and representations of the load-displacement curves from CON, DN, and DN+ES rat tibiae loaded by three-point bending (lower panel). (CON, control; DN, sciatic nerve denervation; DN+ES, sciatic nerve denervation + electrical stimulation).

\section{Discussion}

The current study presents new data on the effects of low-frequency direct ES in the early stages of disuse atrophy in the denervated TA muscles and tibiae of aged rats. The present findings show that ES-induced muscle contraction force mitigates denervation-induced muscle and trabecular bone loss and deterioration of the mechanical properties (e.g., the stiffness, but not the maximal load and elastic modulus) of the tibia mid-diaphysis in the early stages of disuse atrophy in aged rats.

Electrical muscle stimulation affects muscle and bone tissue through various pathways, including the mechanical, circulatory, and humoral pathways. Furthermore, the effects of ES seem to be dependent on the stimulation paradigm, including the intensity, frequency, number of stimuli, and duration of the intervention [3, 5, 19-21]; contradictory reports of the positive and negative effects of ES-induced muscle contraction on disuse osteopenia and muscle loss appear to be due to different ES regimens. In general, relatively high intensities or frequencies of ES are likely to have greater effects on muscle atrophy and bone loss in early-stage DN-induced disuse, due to the higher muscle tension caused by the increased recruitment of muscle fibers and/or the summation of twitch contractions, thereby possibly producing adequate mechanical stimuli to bone. Dynamic muscle stimulation with mid- and high-frequency ES regimens $(20,50$, and $100 \mathrm{~Hz})$ for $10 \mathrm{~min}$ per day for four weeks inhibits trabecular bone loss in the femur (bone volume fraction, trabecular number, trabecular separation, and connection density), as determined by 3D micro-CT analysis in a suspension disuse model of adult rats [7]. Interestingly, the converse has been reported where ES from $1 \mathrm{~Hz}$ to $100 \mathrm{~Hz}$ generates various nonlinear bone stresses and fluid pressure in bone, and that maximal bone strain is observed with
ES at $10 \mathrm{~Hz}$, which mitigates bone loss [22]. Therefore, repetitive twitch muscle contractions might also generate beneficial bone mechanical stress. Previous research using relatively small muscle tensions elicited by ES at $10 \mathrm{~Hz}$, which does not cause tetanic muscle contraction, showed delayed muscle loss in combination with ongoing trabecular bone loss during the early stage of musculoskeletal atrophy due to disuse in young rats $[8,23]$. We applied daily ES at a frequency of $10 \mathrm{~Hz}$ for $2 \mathrm{~s}$ followed by a $6 \mathrm{~s}$ rest, which was repeated for 225 cycles over a period of 30 min. Although this level of ES caused very little twitch contraction summation [8], it is estimated that daily ES treatment would supply 4500 instances of mechanical stimuli per day to the bone. In the present study, this type of ES also had positive effects on reducing trabecular bone volume loss and preventing deterioration of trabecular bone architecture in early-stage disuse musculoskeletal atrophy in aged rats.

The mechanical effects of ES-induced muscle contractions are essential for the maintenance of bone mass and strength, which are achieved through the cooperative functions of osteocytes, osteoblasts, and osteoclasts. Although the mechanisms of the effects of ES on reducing DN-induced trabecular bone loss are still unknown, osteocytes are the principal mechanosensor cells in bone tissue, and may be involved in generating the effects observed in response to low-frequency ES-induced muscle contraction treatment. In a previous study, this type of ES-induced muscle force reduced both trabecular bone loss and the decreases in osteoid and osteocyte numbers embedded in the osteoid area that typically occur following denervation [9]. Additionally, streptomycin treatment did not induce bone loss, but attenuated the effects of ES-induced muscle contractions on reducing disused bone loss in young and aged rats $[9,24]$. Streptomycin treatment also 
resulted in an approximately $70 \%-90 \%$ reduction in the bone anabolic response to ES-induced muscle contractions. Streptomycin is a potent blocker of stretch-activated ion channels, and stretch-activated ion channels are found in osteoblasts and osteocytes. The resulting osteoblastic osteogenesis that is maintained by low-frequency ES-induced muscle contractions may be caused, in part, by activation of the mechanosensors in bone tissue. Thus, the mechanical loading induced by low-frequency ES-induced muscle contractions may have a major influence on the mass and structure of trabecular bone tissue in young and aged rats.

In the present study, our data indicated that low-frequency ES, which does not cause tetanic muscle contraction, also retards trabecular bone loss and structural changes in the early stages of disuse atrophy in aged rats. However, bone strength and the BMD in the cortical bone were not altered by the daily ES-induced muscle contraction treatment regime in aged rats. It has been reported that some differences in metabolic profile and mechano-responsiveness depend on age and bone tissue type, such as whether the bone is trabecular or cortical. First, trabecular bone, with its large surface area, has higher turnover than cortical bone [25]. Trabecular bone is also a type of bone tissue that responds quickly to external factors such as exercise training, and is more metabolically active than cortical bone [26]. At the tissue level, the effects of unloading in the adult rat occur more slowly compared with young rats, and the mechano-responsiveness of the periosteal and endocortical surfaces of cortical bone are approximately 3-11 times smaller in adult and aged tibiae than in young tibiae [13]. Moreover, aging and disuse have been associated with increased osteocyte apoptosis and decreased osteocyte density, which is notable because osteocytes are the principal mechanosensory cells of bone tissue [11]. Osteocyte apoptosis is reportedly a critical determinant of bone strength, and increased osteocyte apoptosis is a common feature in conditions of increased bone fragility [27]. Therefore, could be speculated that the smaller and more gradual effects of ES-induced muscle contraction treatment, which may occur through mechanosensor activation in bone tissue, would be observed in cortical bone in aged rats. In fact, although ES-induced muscle contractions had positive effects on the BMD and bone strength (maximal load) of cortical bone in young rats in our previous study, it did not elicit the same results in aged rats in the present study.

The effects of ES on muscle tissue showed that using a low-frequency ES regimen $(10 \mathrm{~Hz}, 8$ and 16 $\mathrm{mA}, 30 \mathrm{~min} /$ day, for 1 and 3 weeks) in denervated TA muscle retards the atrophy of denervated muscle in young rats $[6,10]$. Reportedly, low-frequency ES (10 $\mathrm{Hz}$ ) also induced the increase in the FCSA in type IIa fibers of adult and aged TA muscle and the number of satellite cells at 1 day, up to 10 days [28, 29]. Satellite cell content is likely to decrease after denervation and also with age; the lower regenerative potential of aged muscles is correlated with declines in satellite cell function. Reportedly, age-related type II muscle fiber atrophy is accompanied by a specific decline in the satellite cell content of type II muscle fibers [30]. Comparatively, increased satellite cell differentiation is correlated with preserved muscle function (muscle force production) in response to electrical stimulation after sciatic nerve injury [31]. Using the same low-frequency ES conditions $(10 \mathrm{~Hz}, 16 \mathrm{~mA}, 30$ min/day, for one week), the present study demonstrated that intervention with direct ES treatment reduces $\mathrm{DN}$-induced decreases in muscle weight, FCSA, and the muscle contraction force of fast-type dominant TA muscles. Therefore, low-frequency ES treatment may also have a beneficial effect on muscle disuse atrophy, which is possibly related to the maintenance of satellite cells in aged rats. Moreover, muscle mass is also an important factor influencing the stimulation delivered to the bone tissue through ES-induced muscle contractions.

The present study has several limitations. First, it is a cross-sectional analysis, and our results observed in aged rats do not reflect longitudinal change over time. We recognize that our results might not be applicable to growing rats undergoing rapid skeletal remodeling; however, the results in the present study can be compared with the results in our previous study using young rats, which was performed using the same protocol as the present study. Secondly, we did not determine the parameters of possible mechanisms that may explain the effects of ES on the reduction of bone loss due to disuse. Thus, further studies are required to elucidate such mechanisms. Although dynamic histomorphometry analyses would increase our understanding of bone formation and bone resorption, the main objective of this study was to assess the effects of low-frequency ES-induced muscle contraction treatment on reducing muscle and bone loss and ameliorating bone fragility in early-stage disuse musculoskeletal atrophy in aged rats. To our knowledge, this is the first study to assess the effects of low-frequency ES-induced muscle contraction on the trabecular bone volume, mechanical properties, and architecture of cortical bone, and the morphological and functional properties of denervated muscles in the early stages of disuse musculoskeletal atrophy in aged rats. Our results may give new and more information about the 
use of ES in denervation situation and osteopenia.

In conclusion, the present study demonstrated that low-frequency ES-induced muscle contraction force retards trabecular bone loss and structural changes, but has little to no impact on cortical bone strength in the early stages of disuse atrophy in aged rats. Also, daily application of low-frequency ES intervention reduces denervation-induced muscle loss and force deficits. The daily ES-induced muscle contraction treatment, which was previously shown to have beneficial effects in young rats, serves the dual purpose of reducing muscle loss and disuse osteopenia in aged rats.

\section{Acknowledgments}

This study was supported in part by a Grant-in-Aid for Scientific Research from the Japan Society for the Promotion of Science (KAKENHI Grant nos. JP16K13021, JP17H02181, and JP17H02125). We thank S. J. Win, PhD, from Edanz Group (www.edanzediting.com/ac) for editing a draft of this manuscript.

\section{Competing Interests}

The authors have declared that no competing interest exists.

\section{References}

1. Kern H, Salmons S, Mayr W, Rossini K, Carraro U. Recovery of long-term denervated human muscles induced by electrical stimulation. Muscle Nerve. 2005;31:98-101.

2. Modlin M, Forstner C, Hofer C, Mayr W, Richter W, Carraro U, Protasi F, Kern $\mathrm{H}$. Electrical stimulation of denervated muscles: first results of a clinical study. Artif Organs. 2005;29:203-6.

3. Dow DE, Dennis RG, Faulkner JA. Electrical stimulation attenuates denervation and age-related atrophy in extensor digitorum longus muscles of old rats. J Gerontol A Biol Sci Med Sci 2005;60:416-24.

4. Dow DE, Cederna PS, Hassett CA, Dennis RG, Faulkner JA. Electrical stimulation prior to delayed reinnervation does not enhance recovery in muscles of rats. Restor Neurol Neurosci. 2007;25:601-10.

5. Gigo-Benato D, Russo TL, Geuna S, Domingues NR, Salvini TF, Parizotto NA. Electrical stimulation impairs early functional recovery and accentuates skeletal muscle atrophy after sciatic nerve crush injury in rats. Muscle Nerve. 2010;41:685-93.

6. Tomori K, Ohta Y, Nishizawa T, Tamaki H, Takekura H. Low-intensity electrical stimulation ameliorates disruption of transverse tubules and neuromuscular junctional architecture in denervated rat skeletal muscle fibers. J Muscle Res Cell Motil. 2010;31:195-205.

7. Lam H, Qin YX. The effects of frequency-dependent dynamic muscle stimulation on inhibition of trabecular bone loss in a disuse model. Bone. 2008;43:1093-100.

8. Tamaki H, Tomori K, Yotani K, Ogita F, Sugawara K, Kirimoto H, Onishi H, Yamamoto N, Kasuga N. Electrical stimulation of denervated rat skeletal muscle retards trabecular bone loss in early stages of disuse musculoskeletal atrophy. J Musculoskelet Neuronal Interact. 2014:14:220-8.

9. Tamaki H, Yotani K, Ogita F, Sugawara K, Kirimoto H, Onishi H, Kasuga N, Yamamoto N. Effect of electrical stimulation-induced muscle force and streptomycin treatment on muscle and trabecular bone mass in early-stage disuse musculoskeletal atrophy. J Musculoskelet Neuronal Interact. 2015;15:270-8.

10. Tamaki H, Yotani K, Ogita F, Hayao K, Nakagawa K, Sugawara K, Kirimoto $\mathrm{H}$, Onishi $\mathrm{H}$, Kasuga N, Yamamoto N. Electrical stimulation of denervated rat skeletal muscle ameliorates bone fragility and muscle loss in early-stage disuse musculoskeletal atrophy. Calcif Tissue Int. 2017;100:420-430.

11. Manolagas SC. Birth and death of bone cells: basic regulatory mechanisms and implications for the pathogenesis and treatment of osteoporosis. Endocr Rev. 2000;21:115-37.
12. Uppuganti S, Granke M, Makowski AJ, Does MD, Nyman JS. Age-related changes in the fracture resistance of male Fischer F344 rat bone. Bone. 2016;83:220-32.

13. Birkhold AI, Razi H, Duda GN, Weinkamer R, Checa S, Willie BM. The Periosteal Bone Surface is Less Mechano-Responsive than the Endocortical. Sci Rep. 2016;6:23480.

14. Kerckhofs G, Durand M, Vangoitsenhoven R, Marin C, Van der Schueren B, Carmeliet G, Luyten FP, Geris L, Vandamme K. Changes in bone macro- and microstructure in diabetic obese mice revealed by high resolution microfocus X-ray computed tomography. Sci Rep. 2016;6:35517.

15. Takekura H, Tamaki H, Nishizawa T, Kasuga N. Plasticity of the transverse tubules following denervation and subsequent reinnervation in rat slow and fast muscle fibres. J Muscle Res Cell Motil. 2003;24:439-51.

16. Bouxsein ML, Boyd SK, Christiansen BA, Guldberg RE, Jepsen KJ, Muller R. Guidelines for assessment of bone microstructure in rodents using micro-computed tomography. J Bone Miner Res. 2010;25:1468-86.

17. Iwamoto J, Takeda T, Katsumata T, Tanaka T, Ichimura S, Toyama Y. Effect of etidronate on bone in orchidectomized and sciatic neurectomized adult rats. Bone. 2002;30:360-7.

18. Brouwers JE, van Rietbergen B, Huiskes R, Ito K. Effects of PTH treatment on tibial bone of ovariectomized rats assessed by in vivo micro-CT. Osteoporos Int. 2009;20:1823-35

19. Midura RJ, Dillman CJ, Grabiner MD. Low amplitude, high frequency strains imposed by electrically stimulated skeletal muscle retards the development of osteopenia in the tibiae of hindlimb suspended rats. Med Eng Phys. 2005;27:285-93

20. Mohr T, Podenphant J, Biering-Sorensen F, Galbo H, Thamsborg G, Kjaer M. Increased bone mineral density after prolonged electrically induced cycle training of paralyzed limbs in spinal cord injured man. Calcif Tissue Int. 1997;61:22-5.

21. Tanaka SM, Yorozuya Y, Takatsu D. Random electromyostimulation promotes osteogenesis and the mechanical properties of rat bones. Ann Biomed Eng. 2017;45:2837-2846.

22. Qin YX, Lam H, Ferreri S, Rubin C. Dynamic skeletal muscle stimulation and its potential in bone adaptation. J Musculoskelet Neuronal Interact. 2010;10:12-24

23. Tamaki H, Yotani K, Ogita F, Kirimoto H, Onishi H, Kasuga N. Bone loss due to disuse and electrical muscle stimulation. J Phys Fitness Sports Med. 2016;5:267-73

24. Tamaki H, Yotani K, Ogita F, Hayao K, Tamakoshi K, Takahashi H, Tsubaki A, Kirimoto H, Onishi H, Kasuga N, Yamamoto N. Streptomycin attenuates the effects of electrical stimulation-induced muscle contraction on reducing trabecular bone loss in the early stages of disuse in old rats. J Bone Miner Res. 2017;32 (Suppl 1):S123.

25. Parfitt AM. Misconceptions (2): turnover is always higher in cancellous than in cortical bone. Bone. 2002;30:807-9.

26. Warner SE, Shea JE, Miller SC, Shaw JM. Adaptations in cortical and trabecular bone in response to mechanical loading with and without weight bearing. Calcif Tissue Int. 2006;79:395-403.

27. Aguirre JI, Plotkin LI, Stewart SA, Weinstein RS, Parfitt AM, Manolagas SC, Bellido T. Osteocyte apoptosis is induced by weightlessness in mice and precedes osteoclast recruitment and bone loss. J Bone Miner Res. 2006;21:605-15.

28. Martins KJ, Murdoch GK, Shu Y, Harris RL, Gallo M, Dixon WT, Foxcroft GR, Gordon T, Putman CT. Satellite cell ablation attenuates short-term fast-to-slow fibre type transformations in rat fast-twitch skeletal muscle. Pflugers Arch. 2009;458:325-35.

29. Putman CT, Sultan KR, Wassmer T, Bamford JA, Skorjanc D, Pette D. Fiber-type transitions and satellite cell activation in low-frequency-stimulated muscles of young and aging rats. J Gerontol A Biol Sci Med Sci. 2001;56:B510-9.

30. Verdijk LB, Snijders T, Drost M, Delhaas T, Kadi F, van Loon LJ. Satellite cells in human skeletal muscle; from birth to old age. Age. 2014·36:545-7.

31. Xing $H$, Zhou M, Assinck P, Liu N. Electrical stimulation influences satellite cell differentiation after sciatic nerve crush injury in rats. Muscle Nerve. 2015;51:400-11. 